\title{
Solution Semicontinuity of Parametric Generalized Vector Equilibrium Problems ${ }^{1}$
}

\author{
C. R. Chen And S. J. $\mathrm{Li}^{2}$ \\ College of Mathematics and Science, \\ Chongqing University, Chongqing, 400030, China \\ E-mails: chencr1981@163.com and lisj@cqu.edu.cn \\ K. L. TEO \\ Department of Mathematics and Statistics, \\ Curtin University of Technology, G.P.O. Box U1987, Perth, WA 6845, Australia \\ E-mail: K.L.Teo@curtin.edu.au
}

October 22, 2008

\begin{abstract}
In this paper, the lower semicontinuity and continuity of the solution mapping to a parametric generalized vector equilibrium problem involving set-valued mappings are established by using a new proof method which is different from the ones used in the literature.
\end{abstract}

Keywords: Lower semicontinuity; Continuity; Solution mappings; Parametric generalized vector equilibrium problems; Scalarization.

\footnotetext{
${ }^{1}$ This research was partially supported by the National Natural Science Foundation of China (Grant numbers: 10871216 and 60574073) and the Natural Science Foundation Project of CQ CSTC (Grant number: 2007BB6117).

${ }^{2}$ Corresponding author.
} 


\section{Introduction}

The vector equilibrium problem is a unified model of several problems, for example, the vector optimization problem, the vector variational inequality problem, the vector complementarity problem and the vector saddle point problem. In the literature, existence results for various types of vector equilibrium problems have been investigated intensively, e.g., see $[7,12]$ and the references therein. The stability analysis of the solution mappings for vector equilibrium problems is another important topic in vector optimization theory. Recently, the semicontinuity, especially the lower semicontinuity, of the solution mappings for parametric vector variational inequalities and parametric vector equilibrium problems has been intensively studied in the literature, such as [5, 6, 8-11,13-21].

Among many approaches for dealing with the lower semicontinuity and continuity of solution mappings for parametric vector variational inequalities and parametric vector equilibrium problems, the scalarization method is of considerable interest. Recently, Cheng and Zhu [11] have obtained a lower semicontinuity result of the solution mapping to a parametric vector variational inequality in finite-dimensional spaces by using a scalarization method. Very recently, by virtue of a density result and scalarization technique, Gong and Yao [13] have first discussed the lower semicontinuity of the efficient solutions for a parametric vector equilibrium problem. By using the ideas of Cheng and Zhu [11], Gong [14] has studied the continuity of the solution mapping for a class of parametric weak vector equilibrium problems in topological vector spaces. Chen and $\mathrm{Li}$ [10] have discussed and improved the lower semicontinuity and continuity results of the efficient and weak efficient solution sets for parametric vector equilibrium problems given in the aforementioned papers [13] and [14], respectively.

Motivated by the work reported in $[5,10,11,14]$, this paper aims to establish the lower semicontinuity and continuity of the solution mapping to a parametric generalized vector equilibrium problem (PGVEP) by using a new proof method which is different from the ones used in [11] and [14]. Our method on lower semicontinuity is based on a scalarization representation of the solution mapping for (PGVEP) and a property involving the union of a family of lower semicontinuous set-valued mappings. Moreover, we show that the sufficient condition which guarantees the lower semicontinuity of the solution mapping is also sufficient for continuity. The upper semicontinuity of the solution mapping is derived by a scalarization method, which is also different from the ones used in the literature. Our consequences are new and include the corresponding results in [11] and [14] as special 
cases.

The rest of the paper is organized as follows. In Section 2, we introduce the problem (PGVEP), and recall some concepts of semicontinuity and their some properties. In Section 3, we discuss the lower semicontinuity and continuity of the solution mapping for (PGVEP).

\section{Preliminaries}

Throughout this paper, let $X$ and $Y$ be real Hausdorff topological vector spaces, and let $Z$ be a real topological space. We also assume that $C$ is a pointed closed convex cone in $Y$ with its interior $\operatorname{int} C \neq \emptyset$. Let $Y^{*}$ be the topological dual space of $Y$ and let $C^{*}:=\left\{f \in Y^{*} \mid f(y) \geq 0, \forall y \in C\right\}$ be the dual cone of $C$.

Suppose that $A$ is a nonempty subset of $X$ and $F: A \times A \rightarrow 2^{Y} \backslash\{\emptyset\}$ is a set-valued mapping. We consider the following generalized vector equilibrium problem (GVEP) of finding $x \in A$ such that

$$
F(x, y) \subset Y \backslash-\operatorname{int} C, \quad \forall y \in A
$$

When the set $A$ and the mapping $F$ are perturbed by a parameter $\mu$ which varies over a set $\Lambda$ of $Z$, we consider the following parametric generalized vector equilibrium problem (PGVEP) of finding $x \in A(\mu)$ such that

$$
F(x, y, \mu) \subset Y \backslash-\operatorname{int} C, \quad \forall y \in A(\mu)
$$

where $A: \Lambda \rightarrow 2^{X} \backslash\{\emptyset\}$ is a set-valued mapping, $F: B \times B \times \Lambda \subset X \times X \times Z \rightarrow 2^{Y} \backslash\{\emptyset\}$ is a set-valued mapping with $A(\Lambda)=\bigcup_{\mu \in \Lambda} A(\mu) \subset B$.

\section{Special Cases.}

(I) Let $\varphi: B \times B \times \Lambda \rightarrow Y$ and $\psi: B \times \Lambda \rightarrow Y$ be vector-valued mappings. Let $F(x, y, \mu)=\varphi(x, y, \mu)+\psi(y, \mu)-\psi(x, \mu)$. Then (PGVEP) reduces to the parameterized weak vector equilibrium problem $(\mathrm{WVEP})_{\mu}$ considered in [14].

(II) Let $X=R^{n}, Y=R^{p}$ and $C=R_{+}^{p}$. Let $g_{i}: B \times \Lambda \rightarrow R^{n}, i=1, \cdots, p$ and $\psi: B \times$ $\Lambda \rightarrow R^{p}$ be vector-valued mappings. Let $F(x, y, \mu)=\left(\left\langle g_{1}(x, \mu), y-x\right\rangle, \cdots,\left\langle g_{p}(x, \mu), y-\right.\right.$ $x\rangle)+\psi(y, \mu)-\psi(x, \mu)$, where $\langle\cdot, \cdot\rangle$ denotes the inner product in the Euclidean space. Then, (PGVEP) also reduces to $(\mathrm{WVVI})_{\mu}$ considered in [14]. Furthermore, let $\psi \equiv 0$. 
Then, (PGVEP) further reduces to the parameterized weak vector variational inequality considered in [11].

For each $\mu \in \Lambda$, let $S(\mu)$ denote the solution set of (PGVEP), i.e.,

$$
S(\mu)=\{x \in A(\mu) \mid F(x, y, \mu) \subset Y \backslash-\operatorname{int} C, \forall y \in A(\mu)\} .
$$

In this paper, by using a new proof method which is different from the ones used in [11] and [14], we will discuss the lower semicontinuity and continuity of $S(\cdot)$ as a set-valued mapping from the set $\Lambda$ into $X$.

Let $\mu \in \Lambda$ and $x \in A(\mu)$. Define $F(x, A(\mu), \mu):=\bigcup_{y \in A(\mu)} F(x, y, \mu)$. Suppose that $\Lambda$ and $\Omega$ are Hausdorff topological spaces and $G: \Lambda \rightarrow 2^{\Omega}$ is a set-valued mapping with nonempty values.

\section{Definition $2.1 \quad[1]$}

(i) $G$ is said to be lower semicontinuous (l.s.c) at $\bar{\lambda} \in \Lambda$ if for any open set $Q \subset \Omega$ with $G(\bar{\lambda}) \cap Q \neq \emptyset$, there exists a neighborhood $N(\bar{\lambda})$ of $\bar{\lambda}$ such that $G(\lambda) \cap Q \neq \emptyset$, for all $\lambda \in N(\bar{\lambda})$.

(ii) $G$ is said to be upper semicontinuous (u.s.c) at $\bar{\lambda}$ if for any open set $Q \subset \Omega$ with $G(\bar{\lambda}) \subset Q$, there exists a neighborhood $N(\bar{\lambda})$ of $\bar{\lambda}$ such that $G(\lambda) \subset Q$, for all $\lambda \in N(\bar{\lambda})$.

We say $G$ is l.s.c (resp. u.s.c) on $\Lambda$, if it is l.s.c (resp. u.s.c) at each $\lambda \in \Lambda$. G is said to be continuous on $\Lambda$ if it is both l.s.c and u.s.c on $\Lambda$.

Proposition 2.1 [1,3]

(i) $G$ is l.s.c at $\bar{\lambda}$ if and only if for any net $\left\{\lambda_{\alpha}\right\} \subset \Lambda$ with $\lambda_{\alpha} \rightarrow \bar{\lambda}$ and any $\bar{x} \in G(\bar{\lambda})$, there exists $x_{\alpha} \in G\left(\lambda_{\alpha}\right)$ such that $x_{\alpha} \rightarrow \bar{x}$.

(ii) If $G$ has compact values (i.e., $G(\lambda)$ is a compact set for each $\lambda \in \Lambda$ ), then $G$ is u.s.c at $\bar{\lambda}$ if and only if for any net $\left\{\lambda_{\alpha}\right\} \subset \Lambda$ with $\lambda_{\alpha} \rightarrow \bar{\lambda}$ and for any $x_{\alpha} \in G\left(\lambda_{\alpha}\right)$, there exist $\bar{x} \in G(\bar{\lambda})$ and a subnet $\left\{x_{\beta}\right\}$ of $\left\{x_{\alpha}\right\}$, such that $x_{\beta} \rightarrow \bar{x}$.

The following lemma plays an important role in the proof of the lower semicontinuity of the solution mapping $S(\cdot)$. 
Lemma 2.1 [2, Theorem 2, p.114] The union $\Gamma=\bigcup_{i \in I} \Gamma_{i}$ of a family of l.s.c set-valued mappings $\Gamma_{i}$ from a topological space $X$ into a topological space $Y$ is also an l.s.c set-valued mapping from $X$ into $Y$, where $I$ is an index set.

\section{Lower Semicontinuity and Continuity}

For each $f \in C^{*} \backslash\{0\}$ and for each $\mu \in \Lambda$, let $S_{f}(\mu)$ denote the set of $f$-efficient solutions to (PGVEP), i.e.,

$$
S_{f}(\mu)=\left\{x \in A(\mu) \mid \inf _{z \in F(x, y, \mu)} f(z) \geq 0, \forall y \in A(\mu)\right\} .
$$

Throughout this section, assume that $S_{f}(\mu) \neq \emptyset$ for all $f \in C^{*} \backslash\{0\}$ and $\mu \in \Lambda$. To ensure the existence, we give a kind of sufficient conditions as an example.

For each $f \in C^{*} \backslash\{0\}$, let $V_{f}$ denote the set of $f$-efficient solutions to (GVEP), i.e.,

$$
V_{f}=\left\{x \in A \mid \inf _{z \in F(x, y)} f(z) \geq 0, \forall y \in A\right\}
$$

A set-valued mapping $E: A \rightarrow 2^{A}$ is called a KKM-mapping if $\operatorname{co}\left\{x_{1}, \cdots, x_{n}\right\} \subset$ $\bigcup_{i=1}^{n} E\left(x_{i}\right)$ for any finite subset $\left\{x_{1}, \cdots, x_{n}\right\}$ of $A$, where $\operatorname{co}(D)$ denotes the convex hull of the set $D$.

The set-valued mapping $G: A \rightarrow 2^{Y}$ is said to be $C$-convex on $A$ if for any $x_{1}, x_{2} \in A$ and $\lambda \in[0,1], \lambda G\left(x_{1}\right)+(1-\lambda) G\left(x_{2}\right) \subset G\left(\lambda x_{1}+(1-\lambda) x_{2}\right)+C$.

Proposition 3.1 Suppose that the following conditions are satisfied:

(i) A is a nonempty compact convex set;

(ii) For each $y \in A, F(\cdot, y)$ is l.s.c on $A$, and for each $x \in A, F(x, \cdot)$ has nonempty compact values on $A$;

(iii) $F(x, x) \subset C$, for all $x \in A$, and $F(x, \cdot)$ is $C$-convex on $A$.

Then, for each $f \in C^{*} \backslash\{0\}, V_{f} \neq \emptyset$.

Proof. Define $M: A \rightarrow 2^{A}$ by

$$
M(y)=\left\{x \in A \mid \inf _{z \in F(x, y)} f(z) \geq 0\right\}, \quad \forall y \in A .
$$


We first prove that for any $y \in A, M(y)$ is a closed set. Let $x_{\alpha} \in M(y)$ and $x_{\alpha} \rightarrow x_{0}$. Then $x_{0} \in A$ since $A$ is compact. Let $g_{y}(x)=\inf _{z \in F(x, y)} f(z)=-\sup _{z \in F(x, y)}(-f(z))$. It follows from $x_{\alpha} \in M(y)$ that

$$
g_{y}\left(x_{\alpha}\right)=\inf _{z \in F\left(x_{\alpha}, y\right)} f(z) \geq 0 .
$$

Since $f$ is continuous and $F(\cdot, y)$ is l.s.c at $x_{0}$, by Proposition 19 of $\left[1, \S 3-1\right.$, p.118], $g_{y}(\cdot)$ is upper semicontinuous at $x_{0}$. It follows from (1) that

$$
\inf _{z \in F\left(x_{0}, y\right)} f(z)=g_{y}\left(x_{0}\right) \geq \limsup _{x_{\alpha} \rightarrow x_{0}} g_{y}\left(x_{\alpha}\right) \geq 0 .
$$

Thus, $x_{0} \in M(y)$. So $M(y)$ is a closed set. Moreover, since $M(y) \subset A$ and $A$ is compact, $M(y)$ is also a compact set.

Next, we show that $M$ is a KKM-mapping. Suppose it is false. Then there exist a finite subset $\left\{y_{1}, \cdots, y_{n}\right\} \subset A$ and $t_{1}, \cdots, t_{n} \geq 0$ with $\sum_{i=1}^{n} t_{i}=1$ such that $\bar{y}=\sum_{i=1}^{n} t_{i} y_{i} \notin$ $\bigcup_{i=1}^{n} M\left(y_{i}\right)$. Then, for each $i \in\{1,2, \cdots, n\}, \bar{y} \notin M\left(y_{i}\right)$, i.e.,

$$
\inf _{z \in F\left(\bar{y}, y_{i}\right)} f(z)<0
$$

Since $f$ is continuous and $F(\bar{y}, \cdot)$ is a compact set, for each $i \in\{1,2, \cdots, n\}$, there exists $z_{i} \in F\left(\bar{y}, y_{i}\right)$ such that

$$
f\left(z_{i}\right)=\min _{z \in F\left(\bar{y}, y_{i}\right)} f(z)<0 .
$$

From the condition (iii), we have

$$
\sum_{i=1}^{n} t_{i} z_{i} \in \sum_{i=1}^{n} t_{i} F\left(\bar{y}, y_{i}\right) \subset F(\bar{y}, \bar{y})+C \subset C+C \subset C .
$$

By the linearity of $f$ and $f \in C^{*} \backslash\{0\}$, we get

$$
\sum_{i=1}^{n} t_{i} f\left(z_{i}\right)=f\left(\sum_{i=1}^{n} t_{i} z_{i}\right) \geq 0
$$

On the other hand, it follows from $t_{i} \geq 0, i=1,2, \cdots, n$, with $\sum_{i=1}^{n} t_{i}=1$ and (2) that

$$
\sum_{i=1}^{n} t_{i} f\left(z_{i}\right)<0
$$

a contradiction. Hence, $M$ is a KKM-mapping.

By the well-known Ky Fan lemma (e.g., see Lemma 2.2 of $[15]), \bigcap_{y \in A} M(y) \neq \emptyset$. Thus, $V_{f}=\bigcap_{y \in A} M(y) \neq \emptyset$.

From Proposition 3.1, we have the following result on the existence of $S_{f}$ readily. 
Proposition 3.2 Let $\mu \in \Lambda$. Suppose that the following conditions are satisfied:

(i) $A(\mu)$ is a nonempty compact convex set;

(ii) For each $y \in A(\mu), F(\cdot, y, \mu)$ is l.s.c on $A(\mu)$, and for each $x \in A(\mu), F(x, \cdot, \mu)$ has nonempty compact values on $A(\mu)$;

(iii) $F(x, x, \mu) \subset C$, for all $x \in A(\mu)$, and $F(x, \cdot, \mu)$ is $C$-convex on $A(\mu)$.

Then, for each $f \in C^{*} \backslash\{0\}, S_{f}(\mu) \neq \emptyset$.

Now we establish the lower semicontinuity and continuity of $S(\cdot)$ to (PGVEP).

Lemma 3.1 For each $\mu \in \Lambda$, if for each $x \in A(\mu), F(x, A(\mu), \mu)+C$ is a convex set, then

$$
S(\mu)=\bigcup_{f \in C^{*} \backslash\{0\}} S_{f}(\mu) .
$$

Proof. "つ" Let $x \in \bigcup_{f \in C^{*} \backslash\{0\}} S_{f}(\mu)$. Then there exists $f^{\prime} \in C^{*} \backslash\{0\}$ such that $x \in S_{f^{\prime}}(\mu)$. Therefore, $x \in A(\mu)$ and $\inf _{z \in F(x, y, \mu)} f^{\prime}(z) \geq 0, \forall y \in A(\mu)$. Whence, we get $\forall y \in A(\mu)$ and $\forall z \in F(x, y, \mu), f^{\prime}(z) \geq 0$, which deduces that $z \notin-\operatorname{int} C$. Otherwise, if $z \in-\operatorname{int} C$, then it follows from $f^{\prime} \in C^{*} \backslash\{0\}$ that $f^{\prime}(z)<0$, a contradiction. Thus, by the arbitrariness of $z$, we obtain $F(x, y, \mu) \subset Y \backslash-\operatorname{int} C, \forall y \in A(\mu)$, and hence $x \in S(\mu)$.

"C" Let $x \in S(\mu)$. Then $x \in A(\mu)$ and $F(x, y, \mu) \subset Y \backslash-\operatorname{int} C, \forall y \in A(\mu)$. Thus,

$$
F(x, A(\mu), \mu) \cap(-\operatorname{int} C)=\emptyset,
$$

and hence,

$$
(F(x, A(\mu), \mu)+C) \cap(-\operatorname{int} C)=\emptyset .
$$

Because $F(x, A(\mu), \mu)+C$ is a convex set, by the well-known Eidelheit's separation theorem (see [4, Theorem 3.16]), there exist a continuous linear functional $f \in Y^{*} \backslash\{0\}$ and a real number $\gamma$ such that

$$
f(\hat{c})<\gamma \leq f(z+c),
$$

for all $z \in F(x, A(\mu), \mu), c \in C$ and $\hat{c} \in-\operatorname{int} C$. Since $C$ is a cone, we get $f(\hat{c}) \leq 0$ for all $\hat{c} \in-\operatorname{int} C$. Thus, $f(\hat{c}) \geq 0$ for all $\hat{c} \in C$, that is, $f \in C^{*}$. Moreover, it follows from $c \in C, \hat{c} \in-\operatorname{int} C$ and the continuity of $f$ that $f(z) \geq 0$ for all $z \in F(x, A(\mu), \mu)$. Thus, $\forall y \in A(\mu)$, we have $\inf _{z \in F(x, y, \mu)} f(z) \geq 0$, i.e., $x \in S_{f}(\mu) \subset \bigcup_{f \in C^{*} \backslash\{0\}} S_{f}(\mu)$. 
Lemma 3.2 Suppose that the following conditions are satisfied:

(i) A is continuous with nonempty compact values on $\Lambda$;

(ii) $F$ is u.s.c with nonempty compact values on $B \times B \times \Lambda$;

(iii) $F(\cdot, \cdot, \mu)$ is $C$-strictly monotone on $A(\mu) \times A(\mu)$ for any given $\mu \in \Lambda$, i.e., for all $x, y \in A(\mu)$ and $x \neq y, F(x, y, \mu)+F(y, x, \mu) \subset-i n t C$.

Then, for each $f \in C^{*} \backslash\{0\}, S_{f}(\cdot)$ is l.s.c on $\Lambda$.

Proof. Suppose to the contrary that there exist $f \in C^{*} \backslash\{0\}$ and $\mu_{0} \in \Lambda$ such that $S_{f}(\cdot)$ is not l.s.c at $\mu_{0}$. Then there exist $\left\{\mu_{\alpha}\right\}$ with $\mu_{\alpha} \rightarrow \mu_{0}$ and $x_{0} \in S_{f}\left(\mu_{0}\right)$, such that for any $x_{\alpha} \in S_{f}\left(\mu_{\alpha}\right), x_{\alpha} \nrightarrow x_{0}$.

Since $A(\cdot)$ is l.s.c at $\mu_{0}$, there exists a net $\bar{x}_{\alpha} \in A\left(\mu_{\alpha}\right)$ such that $\bar{x}_{\alpha} \rightarrow x_{0}$. For any $y_{\alpha} \in S_{f}\left(\mu_{\alpha}\right)$, because $A(\cdot)$ is u.s.c at $\mu_{0}$ with compact values, there exist $y_{0} \in A\left(\mu_{0}\right)$ and a subnet $\left\{y_{\beta}\right\}$ of $\left\{y_{\alpha}\right\}$ such that $y_{\beta} \rightarrow y_{0}$. It follows from $x_{0} \in S_{f}\left(\mu_{0}\right)$ and $y_{0} \in A\left(\mu_{0}\right)$ that

$$
\inf _{z \in F\left(x_{0}, y_{0}, \mu_{0}\right)} f(z) \geq 0 .
$$

Since $f$ is continuous and $F\left(x_{0}, y_{0}, \mu_{0}\right)$ is a compact set, there exists $z_{0 x} \in F\left(x_{0}, y_{0}, \mu_{0}\right)$ such that

$$
f\left(z_{0 x}\right)=\min _{z \in F\left(x_{0}, y_{0}, \mu_{0}\right)} f(z) \geq 0 .
$$

On the other hand, since $y_{\beta} \in S_{f}\left(\mu_{\beta}\right)$ and $\bar{x}_{\beta} \in A\left(\mu_{\beta}\right)$, we get $\inf _{z \in F\left(y_{\beta}, \bar{x}_{\beta}, \mu_{\beta}\right)} f(z) \geq 0$. Since $f$ is continuous and $F\left(y_{\beta}, \bar{x}_{\beta}, \mu_{\beta}\right)$ is a compact set, there exists $z_{\beta} \in F\left(y_{\beta}, \bar{x}_{\beta}, \mu_{\beta}\right)$ such that

$$
f\left(z_{\beta}\right)=\min _{z \in F\left(y_{\beta}, \bar{x}_{\beta}, \mu_{\beta}\right)} f(z) \geq 0 .
$$

Because $F(\cdot, \cdot, \cdot)$ is u.s.c at $\left(y_{0}, x_{0}, \mu_{0}\right)$ with compact values, there exists $z_{0 y} \in F\left(y_{0}, x_{0}, \mu_{0}\right)$ such that $z_{\beta} \rightarrow z_{0 y}$ (taking a subnet if necessary). It follows from the continuity of $f$ and (4) that

$$
f\left(z_{0 y}\right) \geq 0
$$

By (3), (5) and the linearity of $f$, we get

$$
f\left(z_{0 x}+z_{0 y}\right)=f\left(z_{0 x}\right)+f\left(z_{0 y}\right) \geq 0 .
$$


Assume that $y_{0} \neq x_{0}$. Since $F\left(\cdot, \cdot, \mu_{0}\right)$ is $C$-strictly monotone on $A\left(\mu_{0}\right) \times A\left(\mu_{0}\right)$, we have

$$
F\left(x_{0}, y_{0}, \mu_{0}\right)+F\left(y_{0}, x_{0}, \mu_{0}\right) \subset-\operatorname{int} C .
$$

Then it follows from $f \in C^{*} \backslash\{0\}$ and $z_{0 x}+z_{0 y} \in-\operatorname{int} C$ that

$$
f\left(z_{0 x}+z_{0 y}\right)<0
$$

which contradicts (6). Therefore, $y_{0}=x_{0}$. This is impossible by the contradiction assumption.

Theorem 3.1 Suppose that the following conditions are satisfied:

(i) $A$ is continuous with nonempty compact values on $\Lambda$;

(ii) $F$ is u.s.c with nonempty compact values on $B \times B \times \Lambda$;

(iii) $F(\cdot, \cdot, \mu)$ is $C$-strictly monotone on $A(\mu) \times A(\mu)$ for any given $\mu \in \Lambda$;

(iv) For each $\mu \in \Lambda$ and for each $x \in A(\mu), F(x, \cdot, \mu)$ is $C$-convexlike on $A(\mu)$, i.e., for any $x_{1}, x_{2} \in A(\mu)$ and any $\rho \in[0,1]$, there exists $x_{3} \in A(\mu)$ such that $\rho F\left(x, x_{1}, \mu\right)+$ $(1-\rho) F\left(x, x_{2}, \mu\right) \subset F\left(x, x_{3}, \mu\right)+C$.

Then, $S(\cdot)$ is l.s.c on $\Lambda$.

Proof. For each $\mu \in \Lambda$ and for each $x \in A(\mu)$, since $F(x, \cdot, \mu)$ is $C$-convexlike on $A(\mu)$, $F(x, A(\mu), \mu)+C$ is a convex set. Thus, by virtue of Lemma 3.1 , for each $\mu \in \Lambda$, it holds that

$$
S(\mu)=\bigcup_{f \in C^{*} \backslash\{0\}} S_{f}(\mu)
$$

It follows from Lemma 3.2 that for each $f \in C^{*} \backslash\{0\}, S_{f}(\cdot)$ is l.s.c on $\Lambda$. Thus, in view of Lemma 2.1 , we obtain that $S(\cdot)$ is l.s.c on $\Lambda$.

The following example illustrates that the assumption (iii) in Theorem 3.1 is essential.

Example 3.1 Let $X=Z=R, Y=R^{2}, \Lambda=[-1,1]$ and $C=R_{+}^{2}$. Suppose that

$$
A(\mu)=[0,1], F(x, y, \mu)=(\mu x(y-x), x(y-x)), B=[0,1], \text { and } \mu_{0}=0 .
$$


Then the assumptions (i) and (ii) in Theorem 3.1 are clearly satisfied. It can be checked that for each $\mu \in \Lambda$ and for each $x \in A(\mu), F(x, \cdot, \mu)$ is $R_{+}^{2}$-convex on $A(\mu)$, i.e., for every $y_{1}, y_{2} \in A(\mu)$ and $t \in[0,1]$,

$$
t F\left(x, y_{1}, \mu\right)+(1-t) F\left(x, y_{2}, \mu\right) \in F\left(x, t y_{1}+(1-t) y_{2}, \mu\right)+R_{+}^{2} .
$$

Thus, the assumptions (iv) of Theorem 3.1 holds. However, the assumption (iii) in Theorem 3.1 is violated, since for any $x, y \in A\left(\mu_{0}\right)$ and $x \neq y$,

$$
\begin{aligned}
F\left(x, y, \mu_{0}\right)+F\left(y, x, \mu_{0}\right) & =\left(\mu_{0}\left(2 x y-x^{2}-y^{2}\right), 2 x y-x^{2}-y^{2}\right) \\
& =\left(0,2 x y-x^{2}-y^{2}\right) \\
& \notin-i n t R_{+}^{2} .
\end{aligned}
$$

It follows from a direct computation that

$$
S(\mu)= \begin{cases}{[0,1],} & \text { if } \mu \in[-1,0] . \\ \{0\}, & \text { if } \mu \in(0,1],\end{cases}
$$

Clearly, we see that $S(\cdot)$ is not l.s.c at $\mu_{0}=0$. Hence, the assumption (iii) in Theorem 3.1 is essential.

Proposition 3.3 If $F$ has nonempty compact values on $B \times B \times \Lambda$ and $F(\cdot, \cdot, \mu)$ is $C$ strictly monotone on $A(\mu) \times A(\mu)$ for any given $\mu \in \Lambda$, then for each $f \in C^{*} \backslash\{0\}, S_{f}(\cdot)$ is a singleton on $\Lambda$.

Proof. Suppose that there exists $f \in C^{*} \backslash\{0\}$ such that $S_{f}(\cdot)$ is not a singleton on $\Lambda$. Then there exist $\mu \in \Lambda$ and $x_{1}, x_{2} \in S_{f}(\mu)$ satisfying $x_{1} \neq x_{2}$. Thus we have $x_{1}, x_{2} \in A(\mu)$ and

$$
\inf _{z \in F\left(x_{1}, x_{2}, \mu\right)} f(z) \geq 0
$$

and

$$
\inf _{z \in F\left(x_{2}, x_{1}, \mu\right)} f(z) \geq 0 .
$$

Since $f$ is continuous and $F\left(x_{1}, x_{2}, \mu\right)$ is a compact set, there exists $z_{1} \in F\left(x_{1}, x_{2}, \mu\right)$ such that

$$
f\left(z_{1}\right)=\min _{z \in F\left(x_{1}, x_{2}, \mu\right)} f(z) \geq 0 .
$$

Similarly, there exists $z_{2} \in F\left(x_{2}, x_{1}, \mu\right)$ such that

$$
f\left(z_{2}\right)=\min _{z \in F\left(x_{2}, x_{1}, \mu\right)} f(z) \geq 0 .
$$


It follows from $(7),(8)$ and the linearity of $f$ that

$$
f\left(z_{1}+z_{2}\right) \geq 0
$$

By the $C$-strict monotonicity of $F$, we have $z_{1}+z_{2} \in-\operatorname{int} C$. Since $f \in C^{*} \backslash\{0\}, f\left(z_{1}+\right.$ $\left.z_{2}\right)<0$, which leads to a contradiction.

Following the ideas of Cheng and Zhu [11] (see also Gong [14]), we have another proof of Theorem 3.1 based on the fact of Proposition 3.3 as follows.

Another Proof of Theorem 3.1. For each fixed $\mu \in \Lambda$, take arbitrary $x \in S(\mu)=$ $\bigcup_{f \in C^{*} \backslash\{0\}} S_{f}(\mu)$ and $\left\{\mu_{\alpha}\right\}$ with $\mu_{\alpha} \rightarrow \mu$. Then there exists $f^{\prime} \in C^{*} \backslash\{0\}$ such that $\{x\}=$ $S_{f^{\prime}}(\mu)$, because $S_{f^{\prime}}(\mu)$ is a singleton by Proposition 3.3. In view of Lemma 3.2 and Proposition 3.3, $S_{f^{\prime}}(\cdot)$ is continuous at $\mu$, since $S_{f^{\prime}}(\cdot)$ is single-valued. Hence, there exists $\left\{x_{\alpha}\right\}=S_{f^{\prime}}\left(\mu_{\alpha}\right)$ such that $x_{\alpha} \rightarrow x$. Since $x_{\alpha} \in \bigcup_{f \in C^{*} \backslash\{0\}} S_{f}\left(\mu_{\alpha}\right)=S\left(\mu_{\alpha}\right)$, by Proposition 2.1(i), we obtain that $S(\cdot)$ is l.s.c at $\mu$. By the arbitrariness of $\mu \in \Lambda, S(\cdot)$ is l.s.c on $\Lambda$.

Remark 3.1 Our approach on the lower semicontinuity of the solution mapping $S(\cdot)$ is totally different from the ones used by Gong [14] and Cheng and Zhu [11] (cf. the above proof). In our approach, Lemma 2.1 plays an essential role, which allows us to treat $S_{f}$ as a set-valued mapping directly. In fact, because the $C$-strict monotonicity of $F$ is imposed, we see that $S_{f}$ is single-valued (Proposition 3.3), which plays key roles in [14] and [11]. Obviously, our approach does not rely on whether $S_{f}$ is single-valued or not. In addition, compared with [14] and [11], the uniform compactness of $A$ is not required (for more details, see [10]), and the $C$-convexity of $F$ is generalized to the $C$-convexlikeness.

Furthermore, we point out that under the assumptions of Theorem 3.1, the solution mapping $S(\cdot)$ is continuous. We remark that the upper semicontinuity of $S(\cdot)$ is derived by a scalarization method, which is different from the methods with respect to the upper semicontinuity of solution mappings used in the literature, such as $[5,11,14,15,17]$.

Theorem 3.2 Suppose that all conditions of Theorem 3.1 are satisfied. Then, $S(\cdot)$ is continuous on $\Lambda$.

Proof. We shall prove that for each $\mu \in \Lambda, S(\cdot)$ is u.s.c at $\mu$. Suppose that there exists some $\mu_{0} \in \Lambda$ such that $S(\cdot)$ is not u.s.c at $\mu_{0}$. Then there exist an open set $M$ satisfying $S\left(\mu_{0}\right) \subset M$, and nets $\mu_{\alpha} \rightarrow \mu_{0}$ and $x_{\alpha} \in S\left(\mu_{\alpha}\right)$, such that $x_{\alpha} \notin M, \forall \alpha$. 
By Lemma 3.1, we have that $x_{\alpha} \in S\left(\mu_{\alpha}\right)=\bigcup_{f \in C^{*} \backslash\{0\}} S_{f}\left(\mu_{\alpha}\right)$. Thus there exists $f^{\prime} \in C^{*} \backslash\{0\}$ such that $\left\{x_{\alpha}\right\}=S_{f^{\prime}}\left(\mu_{\alpha}\right)$, as $S_{f^{\prime}}\left(\mu_{\alpha}\right)$ is a singleton by Proposition 3.3. Let $\left\{x_{0}\right\}=S_{f^{\prime}}\left(\mu_{0}\right)$. Since $S_{f^{\prime}}(\cdot)$ is continuous at $\mu_{0}$ by Lemma 3.2 and Proposition 3.3, we obatin that $x_{\alpha} \rightarrow x_{0}$. It follows from $x_{\alpha} \notin M$ and the openness of $M$ that $x_{0} \notin M$, which contradicts the fact that $x_{0} \in \bigcup_{f \in C^{*} \backslash\{0\}} S_{f}\left(\mu_{0}\right)=S\left(\mu_{0}\right) \subset M$.

From Theorem 3.2, we have the following corollaries readily. The results improve Theorem 4.2 and Corollary 5.1 of [14], respectively, because the uniform compactness of $A$ is not required.

Corollary 3.1 Let $\psi: B \times \Lambda \rightarrow Y$ and $\varphi: B \times B \times \Lambda \rightarrow Y$ be mappings. Let $F(x, y, \mu)=$ $\varphi(x, y, \mu)+\psi(y, \mu)-\psi(x, \mu)$. Suppose that the following conditions are satisfied:

(i) A is continuous with nonempty compact values on $\Lambda$;

(ii) $\psi$ is continuous on $B \times \Lambda$ and $\varphi$ is continuous on $B \times B \times \Lambda$;

(iii) $\varphi(\cdot, \cdot, \mu)$ is $C$-strictly monotone on $A(\mu) \times A(\mu)$ for any given $\mu \in \Lambda$;

(iv) For each $\mu \in \Lambda$ and for each $x \in A(\mu), \varphi(x, \cdot, \mu)+\psi(\cdot, \mu)$ is $C$-convexlike on $A(\mu)$.

Then, $S(\cdot)$ is continuous on $\Lambda$.

Corollary 3.2 Let $X=R^{n}, Y=R^{p}$ and $C=R_{+}^{p}$. Let $g_{i}: B \times \Lambda \rightarrow R^{n}, i=1, \cdots, p$ and $\psi: B \times \Lambda \rightarrow R^{p}$ be mappings. Let $F(x, y, \mu)=\left(\left\langle g_{1}(x, \mu), y-x\right\rangle, \cdots,\left\langle g_{p}(x, \mu), y-\right.\right.$ $x\rangle)+\psi(y, \mu)-\psi(x, \mu)$. Suppose that the following conditions are satisfied:

(i) A is continuous with nonempty compact values on $\Lambda$;

(ii) $\psi$ and $g_{i}, i=1, \cdots, p$ are continuous on $B \times \Lambda$;

(iii) $g_{i}(\cdot, \mu), i=1, \cdots, p$ are strictly monotone on $A(\mu)$ for any given $\mu \in \Lambda$, i.e., for all $x, y \in A(\mu)$ and $x \neq y,\left\langle g_{i}(x, \mu)-g_{i}(y, \mu), x-y\right\rangle>0, i=1, \cdots, p ;$

(iv) For each $\mu \in \Lambda, \psi(\cdot, \mu)$ is $R_{+}^{p}$-convex on $A(\mu)$.

Then, $S(\cdot)$ is continuous on $\Lambda$. 


\section{Acknowledgment}

The authors would like to thank the anonymous referee for valuable comments and suggestions, which helped to improve the paper.

\section{References}

[1] Aubin, J.P., Ekeland, I.: Applied Nonlinear Analysis. John Wiley and Sons, New York (1984)

[2] Berge, C.: Topological Spaces. Oliver and Boyd, London (1963)

[3] Ferro, F.: A minimax theorem for vector-valued functions. J. Optim. Theory Appl. 60, 19-31 (1989)

[4] Jahn, J.: Vector Optimization-Theory, Applications and Extensions. Springer, Berlin (2004)

[5] Anh, L.Q., Khanh, P.Q.: Semicontinuity of the solution set of parametric multivalued vector quasiequilibrium problems. J. Math. Anal. Appl. 294, 699-711 (2004)

[6] Anh, L.Q., Khanh, P.Q.: On the stability of the solution sets of general multivalued vector quasiequilibrium problems. J. Optim. Theory Appl. 135, 271-284 (2007)

[7] Chen, G.Y., Huang, X.X., Yang, X.Q.: Vector Optimization: Set-Valued and Variational Analysis. Springer, Berlin (2005)

[8] Chen, C.R., Li, S.J.: Semicontinuity of the solution set map to a set-valued weak vector variational inequality. J. Ind. Manag. Optim. 3, 519-528 (2007)

[9] Chen, C.R., Fang, Z.M., Li, S.J.: On the semicontinuity for a parametric generalized vector quasivariational inequality. Dyn. Contin. Discrete Impuls. Syst. Ser. B Appl. Algorithms. (2008) (to appear)

[10] Chen, C.R., Li, S.J.: On the solution continuity of parametric generalized systems. (2008) (submitted)

[11] Cheng, Y.H., Zhu, D.L.: Global stability results for the weak vector variational inequality. J. Global Optim. 32, 543-550 (2005) 
[12] Giannessi, F. (ed.): Vector Variational Inequalities and Vector Equilibria: Mathematical Theories. Kluwer Academic Publishers, Dordrecht (2000)

[13] Gong, X.H., Yao, J.C.: Lower semicontinuity of the set of efficient solutions for generalized systems. J. Optim. Theory Appl. 138, 197-205 (2008)

[14] Gong, X.H.: Continuity of the solution set to parametric weak vector equilibrium problems. J. Optim. Theory Appl. 139, 35-46 (2008)

[15] Huang, N.J., Li, J., Thompson, H.B.: Stability for parametric implicit vector equilibrium problems. Math. Comput. Modelling. 43, 1267-1274 (2006)

[16] Khanh, P.Q., Luu, L.M.: Upper semicontinuity of the solution set to parametric vector quasivariational inequalities. J. Global Optim. 32, 569-580 (2005)

[17] Kimura, K., Yao, J.C.: Sensitivity analysis of solution mappings of parametric vector quasi-equilibrium problems. J. Global Optim. 41, 187-202 (2008)

[18] Kimura, K., Yao, J.C.: Semicontinuity of solution mappings of parametric generalized vector equilibrium problems. J. Optim. Theory Appl. 138, 429-443 (2008)

[19] Li, S.J., Chen, G.Y., Teo, K.L.: On the stability of generalized vector quasivariational inequality problems. J. Optim. Theory Appl. 113, 283-295 (2002)

[20] Li, S.J., Chen, C.R.: Stability of weak vector variational inequality. Nonlinear Anal. DOI 10.1016/j.na.2008.02.032 (2008)

[21] Li, S.J., Fang, Z.M.: On the stability of a dual weak vector variational inequality problem. J. Ind. Manag. Optim. 4, 155-165 (2008) 